\title{
Remarks on BEC on Graphs
}

\author{
Tomohiro Kanda \\ Graduate School of Mathematics, Kyushu University, \\ 744 Motoka, Nishi-ku, Fukuoka 819-0395, JAPAN \\ t-kanda@math.kyushu-u.ac.jp
}

\begin{abstract}
We consider Bose-Einstein condensation (BEC) on graphs with transient adjacency matrix. We prove the equivalence of BEC and non-factoriality of a quasi-free state. Moreover, quasi-free states exhibiting BEC decompose into generalized coherent states. We review necessary and sufficient conditions that a quasi-free state is faithful, factor, and pure and quasi-free states are quasiequivalent, including the paper of H. Araki and M. Shiraishi (1971/72), H. Araki (1971/72), and H. Araki and S. Yamagami (1982). Using their formats and results, we prove necessary and sufficient conditions that a generalized coherent state is faithful, factor, and pure and generalized coherent states are quasiequivalent as well.
\end{abstract}

Keywords: CCR algebra, generalized coherent state, quasi-equivalence, BoseEinstein condensation.

AMS subject classification: $82 \mathrm{~B} 10$ 


\section{Introduction}

In 14, T. Matsui studied the condition for Bose-Einstein condensation (BEC for short.) in terms of the random walk on a graph. In $[\underline{6}$, F. Fidaleo, D. Guido, and T. Isola, in [7 and 8], F. Fidaleo studied some spectral properties of the adjacency matrix of graphs and BEC. They obtained the criterion for BEC on graphs. In [11, J. T. Lewis and J. V. Pulè obtained the non-factoriality of a quasi-free state exhibiting BEC in $L^{2}\left(\mathbb{R}^{3}\right)$ case. However, in case of graphs, BEC implies non-factoriality of a quasi-free states exhibiting BEC is not clear, thus, we study a quasi-free state exhibiting BEC and prove the equivalence of the occurrence of BEC and non-factoriality of a quasi-free state. Moreover, we give factor decomposition of quasi-free states exhibiting BEC into generalized coherent states which are factor and mutually disjoint (Theorem 4.9.). Generalized coherent states are generalization of coherent states in the following sense. Let $\mathfrak{h}$ be a Hilbert space and $\sigma$ be the symplectic form defined by $\sigma(f, g):=\operatorname{Im}\langle f, g\rangle_{\mathfrak{h}}$. In mathematics, a coherent state $\varphi$ on the Weyl CCR algebra $\mathcal{W}(\mathfrak{h}, \sigma)$ is given by

$$
\varphi(W(f))=\exp \left\{-\|f\|_{\mathfrak{h}}^{2} / 4+i \operatorname{Re} \lambda(f)\right\}
$$

for each $f \in \mathfrak{h}$, where $W(f), f \in \mathfrak{h}$, are unitaries which generate $\mathcal{W}(\mathfrak{h}, \sigma)$ and $\lambda$ is a $\mathbb{C}$-linear functional on $\mathfrak{h}$. (See [10, Theorem 3.1.].) A state $\varphi$ on $\mathcal{W}(\mathfrak{h}, \sigma)$ is a generalized coherent state, if there exists a positive semi-definite sesquilinear form $S$ on $\mathfrak{h} \times \mathfrak{h}$ and an $\mathbb{R}$-linear functional $\lambda: \mathfrak{h} \rightarrow \mathbb{R}$ such that

$$
\varphi(W(f))=\exp \{-S(f, f) / 4+i \lambda(f)\}, \quad f \in \mathfrak{h} .
$$

In Section 2, we review works of H. Araki and M. Shiraishi [1, H. Araki 2], and H. Araki and S. Yamagami [3. In [1, H. Araki and M. Shiraishi and in [2, H. Araki considered quasi-free states on the CCR algebra and obtained a condition that a quasi-free state is faithful, factor, and pure. In [3, H. Araki and S. Yamagami got necessary and sufficient conditions that quasi-free states are quasi-equivalent. In [12, J. Manuceau and A. Verbeure and in 13], J. Manuceau, F. Rocca, and D. Testard obtained a condition that a quasi-free state on the Weyl CCR algebra is pure and factor. In [18, A. van Daele obtained conditions of quasi-equivalence of quasi-free states on the Weyl CCR algebra as well. To consider conditions of factoriality, purity and faithfulness of a generalized coherent state and conditions of quasi-equivalence of generalized coherent states in a unified framework, we use formats in [1, 2], and [3.

In Section 3, we consider generalized coherent states on the Weyl CCR algebra. We prove necessary and sufficient conditions that a generalized coherent state is faithful, factor, and pure and necessary and sufficient conditions that generalized coherent states are quasi-equivalent. Moreover, we give an explicit form of factor decomposition of non-factor generalized coherent state. In [9], R. Honegger considered the decomposition of gauge-invariant quasi-free states. In the present paper, we only assume that a state on the Weyl CCR algebra is quasi-free or generalized coherent.

In Section 4 , we review works of F. Fidaleo 8 and consider the non-factoriality of quasi-free states with BEC. We show that a quasi-free state exhibiting BEC is non-factor and such state decomposes into generalized coherent states which are mutually disjoint. In [15, J. V. Pulé, A. F. Verbeure, and V. A. Zagrebnov considered inhomogeneous BEC on $L^{2}\left(\mathbb{R}^{\nu}\right), \nu \geq 1$, and obtained that the 
occurrence of BEC implies spontaneous symmetry breaking and an equilibrium state exhibiting BEC decompose into periodic states. In [14, T. Matsui obtained that the occurrence of BEC implies spontaneous symmetry breaking in case of graphs with some assumptions (See [14, Assumption 1.1.].) as well. In the present paper, generalized coherent states appeared in factor decomposition of a quasi-free state are not periodic. Thus, we give another decomposition of a quasi-free state exhibiting BEC.

\section{Preliminaries}

In this section, we review works of H. Araki and M. Shiraishi [1, H. Araki 2], and H. Araki and S. Yamagami [3. In [1, H. Araki and M. Shiraishi and in [2], $\mathrm{H}$. Araki considered quasi-free states on the CCR algebra and obtained necessary and sufficient conditions that a quasi-free state is factor, pure, and faithful. In 3], H. Araki and S. Yamagami obtained necessary and sufficient conditions that quasi-free states are quasi-equivalent. We use facts presented in this section to consider necessary and sufficient conditions that a generalized coherent state is factor, pure, and faithful and generalized coherent states are quasi-equivalent and to prove non-factoriality of quasi-free states exhibiting BEC.

\subsection{Some Properties of a Quasi-free state}

Let $\tilde{K}$ be a $\mathbb{C}$-linear space and $\gamma_{\tilde{K}}: \tilde{K} \times \tilde{K} \rightarrow \mathbb{C}$ be a sesquilinear form. Let $\Gamma_{\tilde{K}}$ be an anti-linear involution $\left(\Gamma_{\tilde{K}}^{2}=\mathbb{1}\right)$ satisfying $\gamma_{\tilde{K}}\left(\Gamma_{\tilde{K}} f, \Gamma_{\tilde{K}} g\right)=-\gamma_{\tilde{K}}(g, f)$. A CCR algebra $\mathcal{A}\left(\tilde{K}, \gamma_{\tilde{K}}, \Gamma_{\tilde{K}}\right)$ over $\left(\tilde{K}, \gamma_{\tilde{K}}, \Gamma_{\tilde{K}}\right)$ is the quotient of the complex *-algebra generated by $B(f), f \in \tilde{K}$, its adjoint $B(f)^{*}, f \in \tilde{K}$ and an identity over the following relations:

1. $B(f)$ is complex linear in $f$,

2. $B(f)^{*} B(g)-B(g) B(f)^{*}=\gamma_{\tilde{K}}(f, g) \mathbb{1}$,

3. $B\left(\Gamma_{\tilde{K}} f\right)^{*}=B(f)$.

Any linear operator $P$ on $\tilde{K}$ satisfying

1. $P^{2}=P$,

2. $\gamma_{\tilde{K}}(P f, g)>0$, if $P f \neq 0$,

3. $\gamma_{\tilde{K}}(P f, g)=\gamma_{\tilde{K}}(f, P g)$,

4. $\Gamma_{\tilde{K}} P \Gamma_{\tilde{K}}=1-P$,

is called a basis projection.

Let $\mathfrak{h}$ be a complex pre-Hilbert space. A CCR $(*-)$ algebra $\mathcal{A}_{\mathrm{CCR}}(\mathfrak{h})$ over $\mathfrak{h}$ is the quotient of the $*$-algebra generated by $a^{\dagger}(f)$ and $a(f), f \in \mathfrak{h}$, and an identity by the following relations:

1. $a^{\dagger}(f)$ is complex linear in $f$,

2. $\left(a^{\dagger}(f)\right)^{*}=a(f)$, 
3. $\left[a(f), a^{\dagger}(g)\right]=(f, g)_{\mathfrak{h}} \mathbb{1}$ and $\left[a^{\dagger}(f), a^{\dagger}(g)\right]=0=[a(f), a(g)]$.

Let $P$ be a basis projection. Then the mapping $\alpha(P)$ from $\mathcal{A}\left(\tilde{K}, \gamma_{\tilde{K}}, \Gamma_{\tilde{K}}\right)$ to $\mathcal{A}_{\mathrm{CCR}}(P \tilde{K})$ defined by

$$
\begin{aligned}
\alpha(P)\left(B\left(f_{1}\right) B\left(f_{2}\right) \cdots B\left(f_{n}\right)\right) & =\left(\alpha(P) B\left(f_{1}\right)\right)\left(\alpha(P) B\left(f_{2}\right)\right) \cdots\left(\alpha(P) B\left(f_{n}\right)\right) \\
\alpha(P) B(f) & =a^{\dagger}(P f)+a\left(P \Gamma_{\tilde{K}} f\right)
\end{aligned}
$$

is a $*$-isomorphism of $\mathcal{A}\left(\tilde{K}, \gamma_{\tilde{K}}, \Gamma_{\tilde{K}}\right)$ onto $\mathcal{A}_{\mathrm{CCR}}(P \tilde{K})$.

Let $\mathcal{A}$ be a $*$-algebra with identity. A linear functional $\varphi$ on $\mathcal{A}$ is said to be state, if $\varphi$ satisfies $\varphi\left(A^{*} A\right) \geq 0, A \in \mathcal{A}$, and $\varphi(\mathbb{1})=1$. For a state $\varphi$ on $\mathcal{A}$, we have the GNS-representation space $\left(\mathfrak{H}_{\varphi}, \pi_{\varphi}, \xi_{\varphi}\right)$ associated with $\varphi$. We set $\operatorname{Re} \tilde{K}:=\left\{f \in \tilde{K} \mid \Gamma_{\tilde{K}} f=f\right\}$. Then $f \in \operatorname{Re} \tilde{K}$ if and only if $B(f)^{*}=B(f)$.

On $\operatorname{Re} \tilde{K}$, the operators $B(f), f \in \operatorname{Re} \tilde{K}$, correspond to field operators. Moreover, $a^{\dagger}(f)$ and $a(f)$ correspond to the creation operators and the annihilation operators. We give examples of $\tilde{K}, \gamma_{\tilde{K}}$, and $\Gamma_{\tilde{K}}$ in Section 3 and 4

Let $\varphi$ be a state on $\mathcal{A}\left(\tilde{K}, \gamma_{\tilde{K}}, \Gamma_{\tilde{K}}\right)$ such that $\pi_{\varphi}(B(f))$ is essentially selfadjoint for all $f \in \operatorname{Re} \tilde{K}$. Then we put $W_{\varphi}(f)=\exp \left(i \pi_{\varphi}(B(f))\right), f \in \operatorname{Re} \tilde{K}$. Such state $\varphi$ is said to be regular if $W_{\varphi}(f)$ satisfies the Weyl-Segal relations:

$$
W \varphi(f) W_{\varphi}(g)=\exp \left(-\gamma_{\tilde{K}}(f, g) / 2\right) W_{\varphi}(f+g), \quad f, g \in \operatorname{Re} \tilde{K} .
$$

In general, the Weyl CCR algebra is the universal $\mathrm{C}^{*}$-algebra generated by unitaries $W(f), f \in \operatorname{Re} \tilde{K}$, which satisfy 2.2 and we denote $\mathcal{W}\left(\operatorname{Re} \tilde{K}, \gamma_{\tilde{K}}\right)$ the Weyl CCR algebra. (See also [5, Theorem 5.2.8.].)

A state $\varphi$ on $\mathcal{A}\left(\tilde{K}, \gamma_{\tilde{K}}, \Gamma_{\tilde{K}}\right)$ is said to be quasi-free, if $\varphi$ satisfies the following equations:

$$
\begin{aligned}
& \varphi\left(B\left(f_{1}\right) \cdots B\left(f_{2 n-1}\right)\right)=0 \\
& \varphi\left(B\left(f_{1}\right) \cdots B\left(f_{2 n}\right)\right)=\sum \prod_{j=1}^{n} \varphi\left(B\left(f_{s(j)}\right) B\left(f_{s(j+n)}\right)\right),
\end{aligned}
$$

where $n \in \mathbb{N}$ and the sum is over all permutations $s$ satisfying $s(1)<s(2)<$ $\cdots<s(n), s(j)<s(j+n), j=1,2, \cdots, n$. For any quasi-free state $\varphi$ over $\mathcal{A}\left(\tilde{K}, \gamma_{\tilde{K}}, \Gamma_{\tilde{K}}\right)$, the sesquilinear form $S_{\tilde{K}}: \tilde{K} \times \tilde{K} \rightarrow \mathbb{C}$ defined by

$$
S_{\tilde{K}}(f, g)=\varphi\left(B(f)^{*} B(g)\right), \quad f, g \in \tilde{K}
$$

is positive semi-definite and satisfies

$$
\gamma_{\tilde{K}}(f, g)=S_{\tilde{K}}(f, g)-S_{\tilde{K}}(\Gamma g, \Gamma f), \quad f, g \in \tilde{K} .
$$

(See 1, Lemma 3.2.].) Any quasi-free state on $\mathcal{A}\left(\tilde{K}, \gamma_{\tilde{K}}, \Gamma_{\tilde{K}}\right)$ determines the positive semi-definite sesquilinear form $S$, which satisfies the equation 2.5. Conversely, for any positive semi-definite sesquilinear form $S_{\tilde{K}}$ on $\tilde{K} \times \tilde{K}$ satisfying (2.5), there exists a unique quasi-free state $\varphi$ satisfying (2.4) and $\varphi$ is regular. (See [1, Lemma 3.5.].) Thus, there exists a one-to-one correspondence between a positive semi-definite sesquilinear form $S_{\tilde{K}}$ on $\tilde{K} \times \tilde{K}$ and a quasifree state $\varphi$ on $\mathcal{A}\left(\tilde{K}, \gamma_{\tilde{K}}, \Gamma_{\tilde{K}}\right)$. We denote the quasi-free state on $\mathcal{A}\left(\tilde{K}, \gamma_{\tilde{K}}, \Gamma_{\tilde{K}}\right)$ determined by a positive semi-definite sesquilinear form $S_{\tilde{K}}$ by $\varphi_{S}$ defined in 
(2.4). We define the positive semi-definite form $(\cdot, \cdot)_{S}$ on $\tilde{K} \times \tilde{K}$ by the following equation:

$$
(f, g)_{S}:=S_{\tilde{K}}(f, g)+S_{\tilde{K}}\left(\Gamma_{\tilde{K}} g, \Gamma_{\tilde{K}} f\right), \quad f, g \in \tilde{K} .
$$

We set $N_{S}:=\left\{f \in \tilde{K} \mid\|f\|_{S}=0\right\}$, where $\|f\|_{S}=(f, f)_{S}^{1 / 2}$. We denote the completion of $\tilde{K} / N_{S}$ with respect to the norm $\|\cdot\|_{S}$ by $K$. Since $S_{\tilde{K}}(f, f) \leq$ $\|f\|_{S}^{2},\left|\gamma_{\tilde{K}}(f, f)\right| \leq\|f\|_{S}^{2}$, and $\left\|\Gamma_{\tilde{K}} f\right\|_{S}=\|f\|_{S}$ for any $f \in \tilde{K}$, we can extend the sesquilinear form $S_{\tilde{K}}$ and $\gamma_{\tilde{K}}$ to the sesquilinear form on $K \times K$ and the operator $\Gamma_{\tilde{K}}$ to the operator on $K$. We denote the extensions of $S_{\tilde{K}}, \gamma_{\tilde{K}}$, and $\Gamma_{\tilde{K}}$ by $S_{K}, \gamma_{K}$, and $\Gamma_{K}$, respectively. We define the bounded operators $S_{K}$ and $\gamma_{K}$ on $K$ by the following equations:

$$
\begin{aligned}
\left(\xi, S_{K} \eta\right)_{S} & =S_{K}(\xi, \eta), \\
\left(\xi, \gamma_{K} \eta\right)_{S} & =\gamma_{K}(\xi, \eta), \quad \xi, \eta \in K .
\end{aligned}
$$

A quasi-free state $\varphi_{S}$ is said to be Fock type if $N_{S}=\{0\}$ and the spectrum of the operator $S_{K}$ defined in (2.7) is contained in $\{0,1 / 2,1\}$. For any positive semi-definite sesquilinear form $S_{\tilde{K}}$ on $\tilde{K} \times \tilde{K}$, we can construct a Fock type state as follows. Let $\tilde{L}=K \oplus K$. For $\xi_{1}, \xi_{2}, \eta_{1}, \eta_{2} \in K$, we set

$$
\begin{aligned}
\gamma_{L}\left(\xi_{1} \oplus \xi_{2}, \eta_{1} \oplus \widetilde{\left.\eta_{2}\right)}=\right. & \left(\xi_{1}, \gamma_{K} \eta_{1}\right)_{S}-\left(\xi_{2}, \gamma_{K} \eta_{2}\right)_{S}, \\
\widetilde{\Gamma_{L}}= & \Gamma_{K} \oplus \Gamma_{K}, \\
\left(\xi_{1} \oplus \xi_{2}, \eta_{1} \oplus \eta_{2}\right)_{L}= & \left(\xi_{1}, \eta_{1}\right)_{S}+\left(\xi_{2}, \eta_{2}\right)_{S}+2\left(\xi_{1}, S_{K}^{1 / 2}\left(\mathbb{1}-S_{K}\right)^{1 / 2} \eta_{2}\right)_{S} \\
& +2\left(\xi_{2}, S_{K}^{1 / 2}\left(\mathbb{1}-S_{K}\right)^{1 / 2} \eta_{1}\right)_{S} .
\end{aligned}
$$

Let $N_{L}=\left\{\xi \in \tilde{L} \mid(\xi, \xi)_{L}=0\right\}$. Then we denote the completion of $\tilde{L} / N_{L}$ with respect to the norm $\|\cdot\|_{L}$ by $L$. We define the bounded operators $\gamma_{L}$ and $\Pi_{L}$ on $L$ satisfying

$$
\begin{aligned}
\left(\xi, \gamma_{L} \eta\right)_{L} & =\gamma_{L}(\xi, \eta), \quad \xi, \eta \in L . \\
\Pi_{L} & =\frac{1}{2}\left(\mathbb{1}+\gamma_{L}\right) .
\end{aligned}
$$

Then the spectrum of $\Pi_{L}$ on $L$ is contained in $\{0,1 / 2,1\}$. (See [1, Lemma 5.8.] and [1, Lemma 6.1.].) Moreover the following three lemmas hold:

Lemma 2.1. [1, Corollary 6.2.] The map $f \in \tilde{K} \mapsto[f] \in L$, where $[f]:=$ $(f \oplus 0)+N_{L}$, induces a $*$-homomorphism $\alpha_{\tilde{K}}$ of $\mathcal{A}\left(\tilde{K}, \gamma_{\tilde{K}}, \Gamma_{\tilde{K}}\right)$ into $\mathcal{A}\left(L, \gamma_{L}, \Gamma_{L}\right)$. The restriction of a Fock type state $\varphi_{\Pi_{L}}$ of $\mathcal{A}\left(L, \gamma_{L}, \Gamma_{L}\right)$ to $\alpha_{\tilde{K}}\left(\mathcal{A}\left(\tilde{K}, \gamma_{\tilde{K}}, \Gamma_{\tilde{K}}\right)\right)$ gives a quasi-free state $\varphi_{S}$ of $\mathcal{A}\left(\tilde{K}, \gamma_{\tilde{K}}, \Gamma_{\tilde{K}}\right)$ through $\varphi_{\Pi_{L}}\left(\alpha_{\tilde{K}}(A)\right)=\varphi_{S}(A)$.

Lemma 2.2. 2, Lemma 2.3.] Let $R_{S}$ be the von Neumann algebra generated by spectral projections of all $\pi_{\Pi_{L}}(B(f)), f \in \operatorname{Re} \tilde{K}$, on the GNS representation space $\left(\mathfrak{H}_{\Pi_{L}}, \pi_{\Pi_{L}}, \xi_{\Pi_{L}}\right)$ of $\mathcal{A}\left(L, \gamma_{L}, \Gamma_{L}\right)$ associated with $\varphi_{\Pi_{L}}$. Then the following conditions are equivalent:

1. The GNS cyclic vector $\xi_{\Pi_{L}}$ is cyclic for $R_{S}$.

2. The GNS cyclic vector $\xi_{\Pi_{L}}$ is separating for $R_{S}$.

3. The operator $S_{K}$ on $K$ does not have an eigenvalue 0 . 
4. The operator $S_{K}$ on $K$ does not have an eigenvalue 1 .

Lemma 2.3. 2, Lemma 2.4.] The center of $R_{S}$ is generated by $\exp \left(i \pi_{\Pi_{L}}(B(h))\right)$, $h \in \operatorname{Re}\left(\bar{E}_{0} K \oplus 0\right){ }^{L}$, where $E_{0}$ is the spectral projection of $S_{K}$ for $1 / 2$ and ${\overline{\left(E_{0} K \oplus 0\right)}}^{L}$ is the closure of $E_{0} K \oplus 0$ with respect to the norm $\|\cdot\|_{L}$. In particular, $R_{S}$ is factor if and only if $K_{0}=E_{0} K=\{0\}$.

\subsection{Quasi-equivalence of Quasi-free states}

We recall the definitions of quasi-equivalence of representations and states.

Definition 2.4. 2, Definition 6.1.] Let $\pi_{S_{1}}$ and $\pi_{S_{2}}$ be representations associated with quasi-free states $\varphi_{S_{1}}$ and $\varphi_{S_{2}}$ on $\mathcal{A}\left(\tilde{K}, \gamma_{\tilde{K}}, \Gamma_{\tilde{K}}\right)$, respectively. The representations $\pi_{S_{1}}$ and $\pi_{S_{2}}$ are said to be quasi-equivalent, if there exists an isomorphism $\tau$ from $R_{S_{1}}=\left\{W_{S_{1}}(f) \mid f \in \operatorname{Re} \tilde{K}\right\}^{\prime \prime}$ onto $R_{S_{2}}=\left\{W_{S_{2}}(f) \mid f \in \operatorname{Re} \tilde{K}\right\}^{\prime \prime}$ such that

$$
\tau\left(W_{S_{1}}(f)\right)=W_{S_{2}}(f), \quad f \in \operatorname{Re} \tilde{K},
$$

where $W_{S_{1}}(f)=\exp \left(i \pi_{S_{1}}(B(f))\right)$ and $W_{S_{2}}(f)=\exp \left(i \pi_{S_{2}}(B(f))\right)$. Let $\varphi_{S_{1}}$ and $\varphi_{S_{2}}$ be quasi-free states on $\mathcal{A}\left(\tilde{K}, \gamma_{\tilde{K}}, \Gamma_{\tilde{K}}\right)$. The states $\varphi_{S_{1}}$ and $\varphi_{S_{2}}$ are said to be quasi-equivalent, if for each GNS-representations $\left(\mathfrak{H}_{S_{i}}, \pi_{S_{i}}\right), i=1,2$ associated with $\varphi_{S_{i}}$, respectively, are quasi-equivalent.

This definition is equivalent to the definition of quasi-equivalence of states on a $\mathrm{C}^{*}$-algebra. (See [4, Definition 2.4.25.] and [4, Theorem 2.4.26.].)

Let $\varphi_{S_{1}}$ and $\varphi_{S_{2}}$ be quasi-free states on $\mathcal{A}\left(\tilde{K}, \gamma_{\tilde{K}}, \Gamma_{\tilde{K}}\right)$. In [3], H. Araki and S. Yamagami showed the following theorem:

Theorem 2.5. 3, Theorem] Two quasi-free states $\varphi_{S_{1}}$ and $\varphi_{S_{2}}$ on $\mathcal{A}\left(\tilde{K}, \gamma_{\tilde{K}}, \Gamma_{\tilde{K}}\right)$ are quasi-equivalent if and only if the following conditions hold:

1. The topologies induced by $\|\cdot\|_{S_{1}}$ and $\|\cdot\|_{S_{2}}$ are equal.

2. Let $K$ be the completion of $\tilde{K}$ with respect to the topology $\|\cdot\|_{S_{1}}$ or $\|\cdot\|_{S_{2}}$. Then $S_{1}^{1 / 2}-S_{2}^{1 / 2}$ is in the Hilbert-Schmidt class on $K$, where the $S_{1}$ and $S_{2}$ are operators on $K$ defined in (2.7).

\section{$3 \quad$ Generalized Coherent states}

In this section, we consider generalized coherent states on the Weyl CCR algebra. Using facts in the previous section, we give necessary and sufficient conditions that a generalized coherent state is factor, pure, and faithful and generalized coherent states are quasi-equivalent as well.

\subsection{The Weyl CCR algebra}

Let $V$ be an $\mathbb{R}$-linear space with a symplectic form $\sigma: V \times V \rightarrow \mathbb{R}$, i.e., $\sigma$ is a bilinear form on $V$ and satisfy the following relations:

$$
\sigma(f, g)=-\sigma(g, f), \quad f, g \in V .
$$


We assume that there exists an operator $J$ on $V$ with the properties

$$
\sigma(J f, g)=-\sigma(f, J g), \quad J^{2}=-1,
$$

then $V$ is a $\mathbb{C}$-linear space with scalar multiplication defined by

$$
\left(c_{1}+i c_{2}\right) f=c_{1} f+c_{2} J f, \quad c_{1}, c_{2} \in \mathbb{R}, f \in V .
$$

Then we define the complexification $V^{\mathbb{C}}$ of $V$ by $(3.3)$. We set $(f+i g)^{*}=f-i g$ for $f, g \in V$. We fix a symplectic space $(V, \sigma)$ with an operator $J$ satisfying (3.2). We puts $\tilde{K}=V^{\mathbb{C}}$,

$$
\begin{aligned}
\Gamma_{\tilde{K}} f & =f^{*}, \quad f \in \tilde{K}, \\
\gamma_{\tilde{K}}(f, g) & =\frac{1}{2}\left\{\sigma(f, J g)+i \sigma(f, g)-\sigma\left(g^{*}, J f^{*}\right)-i \sigma\left(g^{*}, f^{*}\right)\right\}, \quad f, g \in \tilde{K} 3 .
\end{aligned}
$$

Then on the GNS-representation space $\left(\mathfrak{H}_{\varphi}, \pi_{\varphi}\right)$ associated with a regular state $\varphi$ on $\mathcal{A}\left(\tilde{K}, \gamma_{\tilde{K}}, \Gamma_{\tilde{K}}\right), \mathcal{W}\left(\operatorname{Re} \tilde{K}, \gamma_{\tilde{K}}\right)=\mathcal{W}(V, \sigma)$. Moreover, $\pi_{\varphi}(B(f)), f \in \operatorname{Re} \tilde{K}$, correspond to filed operators. We define the annihilation operators $a(f)$ and the creation operators $a^{\dagger}(f)$ on $\mathfrak{H}_{\varphi}$ by the following equation:

$a_{\varphi}(f):=\left\{\pi_{\varphi}(B(f))+i \pi_{\varphi}(B(i f))\right\} / \sqrt{2}, \quad a^{\dagger}(f):=\left\{\pi_{\varphi}(B(f))-i \pi_{\varphi}(B(i f))\right\} / \sqrt{2}$,

for any $f \in \operatorname{Re} \tilde{K}$.

In this section, we identify the Weyl CCR algebra $\mathcal{W}(V, \sigma)$ with a regular state $\varphi$ and $\mathcal{A}\left(\tilde{K}, \gamma_{\tilde{K}}, \Gamma_{\tilde{K}}\right)$ with $\varphi$, where $\tilde{K}, \gamma_{\tilde{K}}$ and $\Gamma_{\tilde{K}}$ defined in 3.4 .

\subsection{Generalized coherent states}

For an $\mathbb{R}$-linear functional $\lambda: V \rightarrow \mathbb{R}$, there exists a $*$-automorphism $\tau_{\lambda}$ on $\mathcal{W}(V, \sigma)$ defined by

$$
\tau_{\lambda}(W(f)):=e^{i \lambda(f)} W(f), \quad f \in V .
$$

Let $\varphi_{S}$ be a quasi-free state on $\mathcal{W}(V, \sigma)$. Then we define the generalized coherent state $\varphi_{S, \lambda}$ by the following equation:

$$
\varphi_{S, \lambda}(W(f)):=\varphi_{S} \circ \tau_{\lambda}(W(f))=e^{i \lambda(f)} \varphi_{S}(W(f)), \quad f \in V .
$$

We sets $N_{S}=\left\{f \in V^{\mathbb{C}} \mid\|f\|_{S}=0\right\}$, where $\|\cdot\|_{S}=(\cdot, \cdot)_{S}^{1 / 2}$ is the semi-norm defined in (2.6) and $V_{S}^{\mathbb{C}}$ is the completion of $V^{\mathbb{C}} / N_{S}$ by the norm $\|\cdot\|_{S}$. We denote the GNS-representation space with respect to $\varphi_{S}$ and $\varphi_{S, \lambda}$ by $\left(\mathfrak{H}_{S}, \pi_{S}, \xi_{S}\right)$ and $\left(\mathfrak{H}_{S, \lambda}, \pi_{S, \lambda}, \xi_{S, \lambda}\right)$, respectively.

Lemma 3.1. Let $\varphi_{S}$ and $\varphi_{S, \lambda}$ be a quasi-free state and a generalized coherent state on $\mathcal{W}(V, \sigma)$, respectively. Then

$$
R_{S}=R_{S, \lambda},
$$

where $R_{S}$ and $R_{S, \lambda}$ is the von Neumann algebra generated by $\left\{\pi_{S}(W(f)) \mid f \in V\right\}$ and $\left\{\pi_{S, \lambda}(W(f)) \mid f \in V\right\}$, respectively. 
Proof. Since $\varphi_{S}$ is regular, there exist self-adjoint operators $\Psi_{S}(f), f \in V$ such that $\pi_{S}(W(f))=\exp \left(i \Psi_{S}(f)\right)$. By definition of generalized coherent states, we have $\pi_{S, \lambda}(W(f))=e^{i \lambda(f)} \pi_{S}(W(f))$ and $\left(\mathfrak{H}_{S, \lambda}, \pi_{S, \lambda}, \xi_{S, \lambda}\right)=\left(\mathfrak{H}_{S}, \pi_{S, \lambda}, \xi_{S}\right)$. On $\mathfrak{H}_{S}$, we have

$$
\begin{aligned}
& \left\{\pi_{S}(W(f)) \mid f \in V\right\}^{\prime \prime}=\left\{e^{i \lambda(f)} \pi_{S}(W(f)) \mid f \in V\right\}^{\prime \prime} \\
= & \left\{\pi_{S, \lambda}(W(f)) \mid f \in V\right\}^{\prime \prime} .
\end{aligned}
$$

Thus, $R_{S}=R_{S, \lambda}$ by the double commutant theorem.

Theorem 3.2. Let $\varphi_{S, \lambda}$ be a generalized coherent state on $\mathcal{W}(V, \sigma)$. Then $\varphi_{S, \lambda}$ is faithful if and only if $S$ does not have an eigenvalue 0 on $V_{S}^{\mathbb{C}}$.

Proof. Note that $\varphi_{S}$ and $\varphi_{S, \lambda}$ has the same GNS cyclic vector space $\xi_{\Pi_{L}}$. By Lemma 2.2. $\varphi_{S, \lambda}$ is faithful if and only if $S$ does not have an eigenvalue 0 on $V_{S}^{\mathbb{C}}$.

Theorem 3.3. Let $\varphi_{S, \lambda}$ be a generalized coherent state on $\mathcal{W}(V, \sigma)$. Then $\varphi_{S, \lambda}$ is factor if and only if $S$ does not have an eigenvalue $1 / 2$ on $V_{S}^{\mathbb{C}}$.

Proof. By Lemma 2.3 and Lemma 3.1, we have the statement.

Theorem 3.4. Let $(V, \sigma)$ be a non-degenerate symplectic space and $\varphi_{S, \lambda}$ be a generalized coherent state on $\mathcal{W}(V, \sigma)$. Then $\varphi_{S, \lambda}$ is pure if and only if $S$ is a basis projection.

Proof. If $S$ is a basis projection, then by Lemma 3.1 and [1, Lemma 5.5.] $\varphi_{S}$ is pure.

We use the notation in Section 2 Thus, $\tilde{K}=V^{\mathbb{C}}, K=V_{S}^{\mathbb{C}}$, and $L$ is the completion of $V_{S}^{\mathbb{C}} \oplus V_{S}^{\mathbb{C}} / N_{L}$ with respect to the norm $\|\cdot\|_{L}$ defined in 2.11). If $\varphi_{S, \lambda}$ is pure, then by Theorem 3.3. $S$ does not have an eigenvalue $1 / 2$. Then $\Pi_{L}$ defined in 2.13 does not have an eigenvalue $1 / 2$ because the eigenspace of $\Pi_{L}$ with $1 / 2$ is the completion of the set $\left\{f \oplus f \mid f \in E_{0} K\right\}$ with respect to the norm $\|\cdot\|_{L}$, where $E_{0}$ is the spectral projection of $S$ onto $\operatorname{ker}(S-1 / 2)$. (See also the proof of (4) of [1, Lemma 6.1.].) Thus, $\Pi_{L}$ is a basis projection. Using the notation of [1, Lemma 5.5.], we have $R_{S}=R_{\Pi_{L}}\left(H_{1}\right)$, with $H_{1}=$ $[\operatorname{Re} \tilde{K}] \oplus 0 \subset L$ and $\bar{H}_{1}=\overline{\operatorname{Re} K \oplus 0}^{L} \oplus 0$. If $\Pi_{L} \neq S$, then $K \neq L$. Thus, we have $R_{\Pi_{L}}\left(H_{1}\right)^{\prime}=R_{\Pi_{L}}\left(H_{1}^{\perp}\right)$ by [1, Lemma 5.5.] and $H_{1}^{\perp} \neq\{0\}$, where $H_{1}^{\perp}$ is the orthogonal complement with respect to the inner product $(\cdot, \cdot)_{L}$ defined in 2.11). It leads $R_{S}^{\prime} \neq \mathbb{C} \mathbb{1}$. It contradict to the purity of $\varphi_{S}$. Thus, $S$ is a basis projection.

We have necessary and sufficient conditions that a generalized coherent state is faithful, factor, and pure. Next, we consider the quasi-equivalence of generalized coherent states.

Lemma 3.5. Let $\varphi_{S, \lambda}$ be a generalized coherent state on $\mathcal{W}(V, \sigma)$. Then $f \in N_{S}$ if and only if $\pi_{S, \lambda}(W(f))=e^{i \lambda(f)} \mathbb{1}$.

Proof. If $f \in N_{S}$, then $\varphi_{S}(W(t f))=1$ for any $t \in \mathbb{R}$. Thus, by regularity of $\varphi_{S}, \pi_{S}(W(f))=\mathbb{1}$. By definition of generalized coherent state, $\pi_{S, \lambda}(W(f))=$ $e^{i \lambda(f)} \mathbb{1}$.

If $\pi_{S, \lambda}(W(f))=e^{i \lambda(f)} \mathbb{1}, f \in V$, then $\pi_{S}(W(f))=\mathbb{1}$. Since $g^{*}=g$ for any $g \in V$, we have that $(f, f)_{S}=0$. 
Lemma 3.6. Let $\varphi_{S_{1}, \lambda_{1}}$ and $\varphi_{S_{2}, \lambda_{2}}$ be generalized coherent states on $\mathcal{W}(V, \sigma)$. If $\varphi_{S_{1}, \lambda_{1}}$ and $\varphi_{S_{2}, \lambda_{2}}$ are quasi-equivalent, then $N_{S_{1}}=N_{S_{2}}$.

Proof. Since $\varphi_{S_{1}, \lambda_{1}}$ and $\varphi_{S_{2}, \lambda_{2}}$ are quasi-equivalent, then there exists $\tau$ : $\pi_{S_{1}, \lambda_{1}}(\mathcal{W}(V, \sigma))^{\prime \prime} \rightarrow \pi_{S_{2}, \lambda_{2}}(\mathcal{W}(V, \sigma))^{\prime \prime}$ such that

$$
\tau\left(\pi_{S_{1}, \lambda_{1}}(A)\right)=\pi_{S_{2}, \lambda_{2}}(A), \quad A \in \mathcal{W}(V, \sigma) .
$$

If $N_{S_{1}} \neq N_{S_{2}}$, then there exists $f \in V^{\mathbb{C}}$ such that $f \in N_{S_{1}}$ and $f \notin N_{S_{2}}$. Put $h=f+f^{*}$. Then $h \in V=\operatorname{ReV} V^{\mathbb{C}}$ and $h \in N_{S_{1}}$ and $h \notin N_{S_{2}}$. For such $h$, we have

$$
\pi_{S_{1}, \lambda_{1}}(W(h))=e^{i \lambda_{1}(h)} \mathbb{1}
$$

by Lemma 3.5. However, we have

$$
\pi_{S_{2}, \lambda_{2}}(W(h))=e^{i \lambda_{2}(h)} \pi_{2}(W(h))=\tau\left(\pi_{S_{1}, \lambda_{1}}(W(h))\right)=e^{i \lambda_{1}(h)} \mathbb{1} .
$$

It contradict to Lemma 3.5

Theorem 3.7. Let $\varphi_{S_{1}, \lambda_{1}}$ and $\varphi_{S_{2}, \lambda_{2}}$ be generalized coherent states on $\mathcal{W}(V, \sigma)$. Then $\varphi_{S_{1}, \lambda_{1}}$ and $\varphi_{S_{2}, \lambda_{2}}$ are quasi-equivalent if and only if the following conditions hold:

1. $\|\cdot\|_{S_{1}}$ and $\|\cdot\|_{S_{2}}$ induce the same topology,

2. $S_{1}^{1 / 2}-S_{2}^{1 / 2}$ is a Hilbert-Schmidt class operator,

3. $\lambda_{1}=\lambda_{2}$ on $N_{S_{1}}=N_{S_{2}}$,

4. $\lambda_{1}-\lambda_{2}$ is continuous with respect to the norm $\|\cdot\|_{S_{1}}$ or $\|\cdot\|_{S_{2}}$.

Proof. Assume that the topologies induced by $\|\cdot\|_{S_{1}}$ and $\|\cdot\|_{S_{2}}$ are equivalent, $S_{1}^{1 / 2}-S_{2}^{1 / 2}$ is Hilbert-Schmidt class, $\lambda_{1}-\lambda_{2}$ is continuous with respect to $\|\cdot\|_{S_{1}}$, and $\lambda_{1}=\lambda_{2}$ on $N_{S_{1}}=N_{S_{2}}$. Then $\varphi_{S_{1}}$ and $\varphi_{S_{2}}$ are quasi-equivalent by [3, Theorem] and $\varphi_{S_{1}, \lambda_{1}}$ and $\varphi_{S_{2}, \lambda_{2}}$ are quasi-equivalent by continuity of $\lambda_{1}-\lambda_{2}$ and $\lambda_{1}=\lambda_{2}$ on $N_{S_{1}}=N_{S_{2}}$.

Next, we assume that $\varphi_{S_{1}, \lambda_{1}}$ and $\varphi_{S_{2}, \lambda_{2}}$ are quasi-equivalent. The quasiequivalence of $\varphi_{S_{1}, \lambda_{1}}$ and $\varphi_{S_{2}, \lambda_{2}}$ induces the quasi-equivalence of $\varphi_{S_{1}, \lambda_{1}-\lambda_{2}}$ and $\varphi_{S_{2}}$. Put $\lambda:=\lambda_{1}-\lambda_{2}$. Then there exists a $*$-isomorphism $\tau$ from $\pi_{S_{1}, \lambda}(\mathcal{W}(V, \sigma))^{\prime \prime}$ onto $\pi_{S_{2}}(\mathcal{W}(V, \sigma))^{\prime \prime}$ such that

$$
\tau\left(\pi_{S_{1}, \lambda}(A)\right)=\pi_{S_{2}}(A), \quad A \in \mathcal{W}(V, \sigma) .
$$

For any $f \in V$,

$$
\begin{aligned}
& \exp \left(i \lambda(f)-S_{1}(f, f) / 2\right)=\left\langle\xi_{S_{1}}, \tau^{-1}\left(\pi_{S_{2}}(W(f))\right) \xi_{S_{1}}\right\rangle \\
= & \left\langle\xi_{S_{1}}, \tau^{-1}\left(\pi_{S_{2}}(W(f))\right) \xi_{S_{1}}\right\rangle
\end{aligned}
$$

is $\|\cdot\|_{S_{2}}$-continuous in $f \in V$. Thus, $\lambda$ and $S_{1}$ are $\|\cdot\|_{S_{2}}$-continuous. By symmetry, $\lambda$ and $S_{2}$ are $\|\cdot\|_{S_{1}}$-continuous as well. By Lemma 3.5, $N_{S}:=N_{S_{1}}=N_{S_{2}}$. If $\lambda \neq 0$ on $N_{S}$, then there exists $f \in N_{S} \backslash\{0\}$ such that $\lambda(f) \neq 0$. If $\lambda(f)=2 n \pi$ for some $n \in \mathbb{Z}$, then we replace $f$ by $f / \pi$. For such $f$, we have

$$
e^{i \lambda(f)}=\tau\left(\pi_{S_{1}, \lambda}(W(f))\right)=\pi_{S_{2}}(W(f))=\mathbb{1}
$$


by Lemma 3.5. It contradicts to the quasi-equivalence of $\varphi_{S_{1}, \lambda}$ and $\varphi_{S_{2}}$. Thus, $\lambda=0$ on $N_{S}$. Let $\tau^{\prime}$ be the map from $\pi_{S_{1}, \lambda}(\mathcal{W}(V, \sigma))$ to $\pi_{S_{1}}(\mathcal{W}(V, \sigma))$ defined by

$$
\tau^{\prime}\left(\pi_{S_{1}, \lambda}(A)\right)=\pi_{S_{1}}(A), \quad A \in \mathcal{W}(V, \sigma) .
$$

Since $\lambda$ is continuous with respect to the norm $\|\cdot\|_{S_{1}}$ and $\lambda=0$ on $N_{S}$, then we can extend $\tau^{\prime}$ to a map from $\pi_{S_{1}, \lambda}(\mathcal{W}(V, \sigma))^{\prime \prime}$ onto $\pi_{S_{1}}(\mathcal{W}(V, \sigma))^{\prime \prime}$. Then $\tau^{\prime}$ induce the quasi-equivalence of $\varphi_{S_{1}, \lambda}$ and $\varphi_{S_{1}}$. Thus, $\varphi_{S_{1}}$ and $\varphi_{S_{2}}$ are quasiequivalent and by Theorem 2.5 we have the statement.

Remark 3.8. In 20, S. Yamagami obtained quasi-equivalence conditions of (generalized) coherent states in terms of the transition amplitude. For applications to concrete models Hilbert-Schmidt conditions in Theorem 3.7 are easier to handle. Let $\varphi_{S_{1}, \lambda_{1}}$ and $\varphi_{S_{2}, \lambda_{2}}$ be generalized coherent states on the Weyl CCR algebra $\mathcal{W}(V, \sigma)$. Assume that $\varphi_{S_{1}}$ and $\varphi_{S_{2}}$ are quasi-equivalent. If $\lambda_{1}-\lambda_{2}$ is not continuous in $\|\cdot\|_{S_{1}}$ or $\|\cdot\|_{S_{2}}$ or $\lambda_{1} \neq \lambda_{2}$, then the transition amplitude $\left(\varphi_{S_{1}, \lambda_{1}}^{1 / 2}, \varphi_{S_{2}, \lambda_{2}}^{1 / 2}\right)=0$, where $\varphi_{S_{1}}^{1 / 2}$ and $\varphi_{S_{2}, \lambda_{2}}^{1 / 2}$ is GNS-vector in the universal representation space $L^{2}\left(\mathcal{W}(V, \sigma)^{* *}\right)$. (See [20, Theorem 5.3.].)

Factor decompositions of quasi-free states are given in [9, [16 and [19, e.t.c.. For the convenience of the reader, we give an explicit form of factor decomposition of a non-factor generalized coherent state. We recall the definition of the disjointness of states. (See also [4, Definition 4.1.20.] and [4, Lemma 4.2.8.].)

Definition 3.9. Let $\varphi_{1}$ and $\varphi_{2}$ be positive linear functionals on a $\mathrm{C}^{*}$-algebra $\mathcal{A}$. The positive linear functionals $\varphi_{1}$ and $\varphi_{2}$ are said to be disjoint, if for $\omega=\varphi_{1}+\varphi_{2}$, there is a projection $P \in \pi_{\omega}(\mathcal{A})^{\prime \prime} \cap \pi_{\omega}(\mathcal{A})^{\prime}$ such that

$$
\begin{aligned}
& \varphi_{1}(A)=\left(\xi_{\omega}, P \pi_{\omega}(A) \xi_{\omega}\right), \\
& \varphi_{2}(A)=\left(\xi_{\omega},(\mathbb{1}-P) \pi_{\omega}(A) \xi_{\omega}\right), \quad A \in \mathcal{A},
\end{aligned}
$$

where $\pi_{\omega}$ is the GNS-representation and $\xi_{\omega}$ is the GNS-cyclic vector associated with $\omega$.

Note that factor representations are either quasi-equivalent or disjoint. (See e.g. [4, Proposition 2.4.22.], 4, Theorem 2.4.26. (1)], and 4, Proposition 2.4.27.].)

Theorem 3.10. Let $\varphi_{S, \lambda}$ be a generalized coherent state on $\mathcal{W}(V, \sigma)$. If $\varphi_{S, \lambda}$ is non-factor, then there exists a probability measure $\mu$ on $\mathbb{R}^{2 I}$ and $\varphi_{S, \lambda}$ has factor decomposition of the form

$$
\varphi_{S, \lambda}=\int_{\mathbb{R}^{I}} \varphi_{S E_{0}^{\perp}, x \cdot \rho+\lambda} d \mu(x),
$$

where $\varphi_{S E_{0}^{\perp}, x \cdot \rho+\lambda}(W(f))=\exp \left(-S\left(E_{0}^{\perp} f, E_{0}^{\perp} f\right) / 4+i x \cdot \rho(f)+i \lambda(f)\right)$ and $\rho(f)=$ $\left(\operatorname{Re}\left(e_{k}, f\right)_{S}, \operatorname{Im}\left(e_{k}, f\right)_{S}\right)_{k \in I} \in \mathbb{R}^{2 I}$. Moreover, $\varphi_{S E_{0}^{\perp}, x \cdot \rho+\lambda}$ and $\varphi_{S E_{0}^{\perp}, y \cdot \rho+\lambda}$ are disjoint unless $x \neq y, x, y \in \mathbb{R}^{2 I}$.

Proof. If a generalized coherent state $\varphi_{S, \lambda}$ on $\mathcal{W}(V, \sigma)$ is non-factor, then on $V_{S}^{\mathbb{C}}, S$ has the spectral decomposition

$$
S f=S E_{0}^{\perp} f+\frac{1}{2} \sum_{k \in I}\left(e_{k}, f\right)_{S} e_{k}, \quad f \in V_{S}^{\mathbb{C}},
$$


where $E_{0}$ is the spectral projection of $S$ with an eigenvalue $1 / 2, I$ is an index set such that $|I|=\operatorname{dim} \operatorname{ker}(S-1 / 2)$, and $\left\{e_{k}\right\}_{k \in I}$ is an orthonormal basis for $\operatorname{ker}(S-1 / 2)$. Thus, for any $W(f), f \in V$, we have

$$
\varphi_{S, \lambda}(W(f))=\exp \left(-\frac{S\left(E_{0}^{\perp} f, E_{0}^{\perp} f\right)}{4}+i \lambda(f)\right) \exp \left(-\frac{\sum_{k \in I}\left|\left(e_{k}, f\right)_{S}\right|^{2}}{8}\right) .
$$

By a theorem of Bochner-Minlos (See e.g. 17, Theorem 2.2.]), there exists a probability measure $\mu$ on $\mathbb{R}^{2 I}$ such that

$$
\exp \left(-\frac{\sum_{k}\left|\left(e_{k}, f\right)_{S}\right|^{2}}{8}\right)=\int_{\mathbb{R}^{2 I}} \exp (i x \cdot \rho(f)) d \mu(x),
$$

where $\rho(f)=\left(\operatorname{Re}\left(e_{k}, f\right)_{S}, \operatorname{Im}\left(e_{k}, f\right)_{S}\right)_{k \in I} \in \mathbb{R}^{2 I}$. For $\varphi_{S E_{0}^{\perp}, x \cdot \rho+\lambda}$, we have $N_{S E_{\circ}^{\perp}}=E_{0} V^{\mathbb{C}} \neq\{0\}$. Since $E_{0} V^{\mathbb{C}} \neq\{0\}$, there exists a $f \in V$ such that $\operatorname{Re}\left(e_{k}, f\right)_{S} \neq 0$ or $\operatorname{Im}\left(e_{k}, f\right)_{S} \neq 0$. We put $f_{n}:=E_{0} f+1 / n E_{0}^{\perp} f$. Then $\left\|f_{n}\right\|_{S E_{0}^{\perp}} \rightarrow 0$ and $\operatorname{Re}\left(e_{k}, f_{n}\right)_{S} \not \rightarrow 0$ or $\operatorname{Im}\left(e_{k}, f_{n}\right)_{S} \not \rightarrow 0$ as $n \rightarrow \infty$. Thus, the generalized coherent states $\varphi_{S E_{0}^{\perp}, x \cdot \rho+\lambda}$ and $\varphi_{S E_{0}^{\perp}, y \cdot \rho+\lambda}, x, y \in \mathbb{R}^{2 I}$ are not quasi-equivalent unless $x=y$ by Theorem 3.7. Since $\|\cdot\|_{S}$ and $\|\cdot\|_{S E_{0}^{\perp}}$ induce the same topology on $V^{\mathbb{C}}$ and $S E_{0}^{\perp}$ on $V_{S E_{0}^{\perp}}^{\mathbb{C}}$ does not have an eigenvalue $1 / 2$, $\varphi_{S E_{0}^{\perp}, x \cdot \rho+\lambda}$ is factor and $\varphi_{S E_{0}^{\perp}, x \cdot \rho+\lambda}$ and $\varphi_{S E_{0}^{\perp}, y \cdot \rho+\lambda}$ are disjoint unless $x \neq y$, $x, y \in \mathbb{R}^{2 I}$.

\section{BEC and Non-factor states}

In this section, we consider quasi-free states on $\mathcal{W}(\mathfrak{h}, \sigma)$, where $\mathfrak{h}$ is a pre-Hilbert space over $\mathbb{C}$ with an inner product $\langle\cdot, \cdot\rangle_{\mathfrak{h}}$ and $\sigma(f, g)=\operatorname{Im}\langle f, g\rangle_{\mathfrak{h}}, f, g \in \mathfrak{h}$. We give the decomposition of quasi-free states on $\mathcal{W}(\mathfrak{h}, \sigma)$ into generalized coherent states which are mutually disjoint.

\subsection{General properties}

In this subsection, we use the following notations. Let $\mathfrak{h}$ be a subspace of a Hilbert space over $\mathbb{C}$. We assume that $\mathfrak{h}$ is equipped with positive definite inner products $\langle\cdot, \cdot\rangle_{\mathfrak{h}}$ and $\langle\cdot, \cdot\rangle_{0}$. Let $q$ be a linear functional on $\mathfrak{h}$. We consider the quasi-free state $\varphi_{q, D}, D \geq 0$, on $\mathcal{W}(\mathfrak{h}, \sigma)$ defined by

$$
\varphi_{q, D}\left(a^{\dagger}(f) a(g)\right)=\langle g, f\rangle_{0}+D \overline{q(g)} q(f),
$$

where $a(f)$ and $a^{\dagger}(f), f \in \mathfrak{h}$, are the annihilation operators and the creation operators on the GNS representation space $\mathfrak{H}_{\varphi_{q, D}}$, respectively. Note that the annihilation operators $a(f), f \in \mathfrak{h}$, and the creation operators $a^{\dagger}(f), f \in \mathfrak{h}$ satisfy the following equation:

$$
\left[a(f), a^{\dagger}(g)\right]=\langle f, g\rangle_{\mathfrak{h}}, \quad[a(f), a(g)]=0=\left[a^{\dagger}(f), a^{\dagger}(g)\right], \quad f, g \in \mathfrak{h} .
$$

Our aim is to show that $\varphi_{q, D}$ is non-factor if $q$ is not continuous with respect to the norm $\|\cdot\|_{q, D}$ defined in $(4.8)$ and $D>0$, and to get factor decomposition 
of $\varphi_{q, D}$, in this subsection. Let $\left\{e_{n}\right\}_{n \in \mathbb{N}}$ be an orthonormal basis on a Hilbert space which is contained in $\mathfrak{h}$. Fix $\left\{e_{n}\right\}_{n \in \mathbb{N}}$. We set

$$
\bar{f}=\sum_{n \in \mathbb{N}} \overline{f_{n}} e_{n}
$$

for $f=\sum_{n \in \mathbb{N}} f_{n} e_{n} \in \mathfrak{h}$, where $f_{n} \in \mathbb{C}, n \in \mathbb{N}$ and $\overline{f_{n}}$ is the complex conjugate of $f_{n}$. For a linear functional $q$ and $D \geq 0$, we put $\tilde{K}_{q, D}=\mathfrak{h} \oplus \mathfrak{h}$. For $f_{1}, f_{2}, g_{1}, g_{2} \in$ $\mathfrak{h}$, we sets

$$
\begin{aligned}
& \gamma_{D}\left(f_{1} \oplus f_{2}, g_{1} \oplus g_{2}\right)=\frac{1}{2}\left(\left\langle f_{1}, g_{1}\right\rangle_{\mathfrak{h}}-\left\langle f_{2}, g_{2}\right\rangle_{\mathfrak{h}}\right), \\
& \Gamma\left(f_{1} \oplus f_{2}\right)=\overline{f_{2}} \oplus \overline{f_{1}}, \\
& B\left(f_{1} \oplus f_{2}\right)=\frac{1}{\sqrt{2}}\left(a^{\dagger}\left(f_{1}\right)+a\left(\overline{f_{2}}\right)\right), \\
& S_{q, D}\left(f_{1} \oplus f_{2}, g_{1} \oplus g_{2}\right)=\varphi_{q, D}\left(B\left(f_{1} \oplus f_{2}\right)^{*} B\left(g_{1} \oplus g_{2}\right)\right) \\
= & \frac{1}{2} \varphi_{q, D}\left(\left(a^{\dagger}\left(f_{1}\right)+a\left(\overline{f_{2}}\right)\right)^{*}\left(a^{\dagger}\left(g_{1}\right)+a\left(\overline{g_{2}}\right)\right)\right) \\
= & \frac{1}{2}\left\langle f_{1}, g_{1}\right\rangle_{\mathfrak{h}}+\frac{1}{2}\left\langle f_{1}, g_{1}\right\rangle_{0}+\frac{1}{2}\left\langle f_{2}, g_{2}\right\rangle_{0}+\frac{D}{2} \overline{q\left(f_{1}\right)} q\left(g_{1}\right)+\frac{D}{2} \overline{q\left(f_{2}\right)} q\left(g_{2}\langle 4.7)\right.
\end{aligned}
$$

We define the inner product on $\tilde{K}_{q, D}$ by

$$
\begin{aligned}
\left\langle f_{1} \oplus f_{2}, g_{1} \oplus g_{2}\right\rangle_{q, D}= & \frac{1}{2}\left\langle f_{1}, g_{1}\right\rangle_{\mathfrak{h}}+\frac{1}{2}\left\langle f_{2}, g_{2}\right\rangle_{\mathfrak{h}}+\left\langle f_{1}, g_{1}\right\rangle_{0}+\left\langle f_{2}, g_{2}\right\rangle_{0} \\
& +D \overline{q\left(f_{1}\right)} q\left(g_{1}\right)+D \overline{q\left(f_{2}\right)} q\left(g_{2}\right) .
\end{aligned}
$$

Let $N_{K_{q, D}}=\left\{f \in \tilde{K}_{q, D} \mid\|f\|_{q, D}=0\right\}$. Then we denote the completion of $\tilde{K}_{q, D} / N_{K_{q, D}}$ with respect to the norm $\|\cdot\|_{q, D}$ by $K_{q, D}$. In this case, $\left\|f_{1} \oplus f_{2}\right\|_{q, D}=$ 0 leads $f_{1}=0$ and $f_{2}=0$. Thus, $N_{K_{q, D}}=\{0\}$.

We put

$$
\langle f, g\rangle_{\mathfrak{K}}=\frac{1}{2}\langle f, g\rangle_{\mathfrak{h}}+\langle f, g\rangle_{0}, \quad f, g \in \mathfrak{h},
$$

and $\|\cdot\|_{\mathfrak{K}}=\langle\cdot, \cdot\rangle_{\mathfrak{K}}^{1 / 2}$. We define the Hilbert space $\mathfrak{K}$ by the completion of $\mathfrak{h}$ with respect to the norm $\|\cdot\|_{\mathfrak{K}}$.

Lemma 4.1. The space $K_{q, D}$ has the following form:

1. If $D>0$ and $q$ is not continuous with respect to the norm $\|\cdot\|_{\mathfrak{K}}$, then we have

$$
K_{q, D}=\mathbb{C} \oplus \mathfrak{K} \oplus \mathbb{C} \oplus \mathfrak{K},
$$

2. If $D=0$ or $q$ is continuous with respect to the norm $\|\cdot\|_{\mathfrak{K}}$, then we have

$$
K_{q, D}=\mathfrak{K} \oplus \mathfrak{K} .
$$

Proof. We consider the case of $D>0$ and $q$ is not continuous with respect to the norm $\|\cdot\|_{\mathfrak{K}}$. It suffices to show that $\mathbb{C} \oplus \mathfrak{K}=\overline{\mathfrak{h}}$, where $\overline{\mathfrak{h}}$ is the completion of $\mathfrak{h}$ with respect to the norm $\|\cdot\|^{\prime}$ defined by

$$
\left(\|f\|^{\prime}\right)^{2}=\|f\|_{\mathfrak{K}}^{2}+D|q(f)|^{2}, \quad f \in \mathfrak{h}
$$


We define $\pi: \mathfrak{h} \rightarrow \mathbb{C} \oplus \mathfrak{K}$ by

$$
\pi(f)=q(f) \oplus f
$$

Since $q$ is not continuous, for any $f \in \mathfrak{h}$, there exists a sequence $f_{n}$ in $\mathfrak{h}$ such that $\lim _{n \rightarrow \infty}\left\|f_{n}-f\right\|_{\mathfrak{K}}=0$ and $\lim _{n \rightarrow \infty} q\left(f_{n}\right)=0$. For such $f_{n}$ and $f$, we have

$$
\pi\left(f_{n}-f\right) \rightarrow q(f) \oplus 0, \quad \pi\left(f_{n}\right) \rightarrow 0 \oplus f .
$$

The case of $D=0$ is clear. We assume that $q$ is continuous with respect to the norm $\|\cdot\|_{\mathfrak{K}}$. By continuity of $q$, the norm $\|\cdot\|^{\prime}$, defined in 4.12 , and $\|\cdot\|_{\mathfrak{K}}$ induce the same topology.

Theorem 4.2. For a linear space $\mathfrak{h}$ with positive definite inner products $\langle\cdot, \cdot\rangle_{\mathfrak{h}}$ and $\langle\cdot, \cdot\rangle_{0}$, if $D>0$ and $q$ is not continuous with respect to the norm $\|\cdot\|_{\mathfrak{K}}$, then the two-point function $\varphi_{q, D}$ defined in 4.1) is a non-factor state on $\mathcal{W}(\mathfrak{h}, \sigma)$.

Proof. First, we consider the case of $D>0$. By Lemma 2.1 and Lemma 2.2 it suffices to show that $1 / 2 \in \sigma_{P}\left(S_{q, D}\right)$. By Lemma 4.1. an element of $K_{q, D}$ has the form $\left(a_{1}, f_{1}, a_{2}, f_{2}\right), a_{1}, a_{2} \in \mathbb{C}, f_{1}, f_{2} \in \mathfrak{K}$. For any $\left(a_{1} \oplus f_{1} \oplus a_{2} \oplus f_{2}\right),(b \oplus$ $0 \oplus 0 \oplus 0) \in K_{q, D}, b \in \mathbb{C}$, the operator $S_{q, D}$ satisfies

$$
\begin{aligned}
& \left\langle\left(a_{1} \oplus f_{1} \oplus a_{2} \oplus f_{2}\right), S_{q, D}\left(b_{1} \oplus 0 \oplus 0 \oplus 0\right)\right\rangle_{q, D}=\frac{D}{2} \overline{a_{1}} b \\
= & \frac{1}{2}\left\langle\left(a_{1} \oplus f_{1} \oplus a_{2} \oplus f_{2}\right),(b \oplus 0 \oplus 0 \oplus 0)\right\rangle_{q, D} .
\end{aligned}
$$

Thus, we have $S_{q, D}(b \oplus 0 \oplus 0 \oplus 0)=1 / 2(b \oplus 0 \oplus 0 \oplus 0)$ for any $b \in \mathbb{C}$ and $1 / 2 \in \sigma_{P}\left(S_{q, D}\right)$.

Proposition 4.3. For a linear space $\mathfrak{h}$ with positive definite inner products $\langle\cdot, \cdot\rangle_{\mathfrak{h}}$ and $\langle\cdot, \cdot\rangle_{0}$, if $D=0$ or $q$ is continuous with respect to the norm $\|\cdot\|_{\mathfrak{K}}$, the two-point function $\varphi_{q, D}$ defined in (4.1) is a factor state on $\mathcal{W}(\mathfrak{h}, \sigma)$.

Proof. If $q$ is continuous with respect to the norm $\|\cdot\|_{\mathfrak{K}}$, then $\varphi_{q, D}$ is quasiequivalent to $\varphi_{0,0}$ by Theorem 3.7. Thus, it suffice to show the case of $D=0$. There exists the positive contraction operator $A$ on $\mathfrak{K}$ such that $\langle\xi, A \eta\rangle_{\mathfrak{K}}=$ $\langle\xi, \eta\rangle_{\mathfrak{h}} / 2$ and $\langle\xi,(\mathbb{1}-A) \eta\rangle_{\mathfrak{K}}=\langle\xi, \eta\rangle_{0}, \xi, \eta \in \mathfrak{K}$. Then $S_{0,0}$ has the following form:

$$
S_{0,0}\left(\eta_{1} \oplus \eta_{2}\right)=(A+(\mathbb{1}-A) / 2) \eta_{1} \oplus \frac{\mathbb{1}-A}{2} \eta_{2}=\frac{\mathbb{1}+A}{2} \eta_{1} \oplus \frac{\mathbb{1}-A}{2} \eta_{2},
$$

for $\eta_{1}, \eta_{2} \in \mathfrak{K}$. If $1 / 2 \in \sigma_{P}\left(S_{0,0}\right)$, then $(\mathbb{1}+A) \eta_{1}=\eta_{1}$ and $(\mathbb{1}-A) \eta_{2}=\eta_{2}$. Thus, $\eta_{1}, \eta_{2} \in \operatorname{ker} A$. Since the positive definiteness of $\langle\cdot, \cdot\rangle_{\mathfrak{h}}$ and $\langle\cdot, \cdot\rangle_{0}$ on $\mathfrak{h}$, $\mathfrak{h} \cap \operatorname{ker} A=\{0\}$. Thus, $\operatorname{ker} A=\{0\}$ and $\varphi_{0,0}$ is factor.

Next, we consider factor decomposition of $\varphi_{q, D}$, if $q$ is not continuous in $\|\cdot\|_{\mathfrak{K}}$. Let $\left(\mathfrak{H}_{0}, \pi_{0}, \xi_{0}\right)$ be the GNS-representation space with respect to $\varphi_{0}:=\varphi_{q, 0}=$ $\varphi_{0, D}$. Since $\varphi_{0}$ is regular state on $\mathcal{W}(\mathfrak{h}, \sigma)$, there exist self-adjoint operators $\Psi_{0}(f), f \in \mathfrak{h}$, such that

$$
\pi_{0}(W(f))=\exp \left(i \Psi_{0}(f)\right)
$$

Now we define the field operators $\Psi_{s_{1}, s_{2}}(f), s_{1}, s_{2} \in \mathbb{R}, f \in \mathfrak{h}$, on $\mathfrak{H}_{0}$ by

$$
\Psi_{s_{1}, s_{2}}(f)=\Psi_{0}(f)+s_{1} D^{1 / 2} \operatorname{Re} q(f) \mathbb{1}+s_{2} D^{1 / 2} \operatorname{Im} q(f) \mathbb{1}, \quad f \in \mathfrak{h} .
$$


Let $\pi_{s_{1}, s_{2}}$ be the representation of $\mathcal{W}(\mathfrak{h}, \sigma)$ on $\mathfrak{H}_{0}$ defined by

$$
\pi_{s_{1}, s_{2}}(W(f))=\exp \left(i \Psi_{s_{1}, s_{2}}(f)\right), \quad f \in \mathfrak{h} .
$$

Using the $\pi_{s_{1}, s_{2}}$, we define the state $\varphi_{s_{1}, s_{2}}$ on $\mathcal{W}(\mathfrak{h}, \sigma)$ by

$$
\varphi_{s_{1}, s_{2}}(A)=\left\langle\xi_{0}, \pi_{s_{1}, s_{2}}(A) \xi_{0}\right\rangle, \quad A \in \mathcal{W}(\mathfrak{h}, \sigma) .
$$

Then we have the following theorem.

Theorem 4.4. If $q$ is not continuous in $\|\cdot\|_{\mathfrak{K}}$, then for each $s_{1}, s_{2}, t_{1}, t_{2} \in \mathbb{R}$, $\varphi_{s_{1}, s_{2}}$ and $\varphi_{t_{1}, t_{2}}$ are factor and disjoint unless $t_{1}=s_{1}$ and $t_{2}=s_{2}$.

Proof. By Lemma 3.1 and Proposition 4.3. $\varphi_{s_{1}, s_{2}}$ and $\varphi_{t_{1}, t_{2}}$ are factor. Since $q$ is not continuous with respect to the norm, $\varphi_{s_{1}, s_{2}}$ and $\varphi_{t_{1}, t_{2}}$ are disjoint unless $t_{1}=s_{1}$ and $t_{2}=s_{2}$ by Theorem 3.7 .

Finally, we obtain factor decomposition of $\varphi_{q, D}$.

Theorem 4.5. If $q$ is not continuous in $\|\cdot\|_{\mathfrak{K}}$, then for any $D>0$, factor decomposition of $\varphi_{q, D}$ defined in 4.1] is given by

$$
\varphi_{q, D}=\frac{1}{2 \pi} \int_{\mathbb{R}^{2}} \varphi_{s_{1}, s_{2}} e^{-\frac{s_{1}^{2}+s_{2}^{2}}{2}} d s_{1} d s_{2} .
$$

Proof. By Theorem 3.10, we are done.

\subsection{On graphs}

In this subsection, let $X=(V X, E X)$ be an undirected graph, where $V X$ is the set of all vertices in $X$ and $E X$ is the set of all edges in $X$. Two vertices $x, y \in V X$ are said to be adjacent if there exists an edge $(x, y) \in E X$ joining $x$ and $y$, and we write $x \sim y$. We denote the set of all the edges connecting $x$ with $y$ by $E_{x, y}$. Since the graph is undirected, $E_{x, y}=E_{y, x}$. Let $\ell^{2}(V X)$ be the set of all square summable sequence labeled by the vertices in $V X$. Let $A_{X}$ be the adjacency operator of $X$ defined by

$$
\left\langle\delta_{x}, A_{X} \delta_{y}\right\rangle=\left|E_{x, y}\right|, \quad x, y \in V X .
$$

In addition, for any $x \in V X$, we set the degree of $x$ by $\operatorname{deg}(x)$ and

$$
\operatorname{deg}:=\sup _{x \in V X} \operatorname{deg}(x) .
$$

We assume that $X$ is connected, countable and $\operatorname{deg}<\infty$. Then, the adjacency operator $A_{X}$ acting on $\ell^{2}(V X)$ is bounded. If for any $\delta_{x}, x \in V X, A_{X}$ satisfies the condition

$$
\lim _{\lambda \searrow\left\|A_{X}\right\|}\left\langle\delta_{x},\left(\lambda \mathbb{1}-A_{X}\right)^{-1} \delta_{x}\right\rangle<\infty,
$$

then $A_{X}$ is said to be transient. Let $H$ be the Hamiltonian on $\ell^{2}(V X)$ defined by $H:=\left\|A_{X}\right\| \mathbb{1}-A_{X}$.

A bounded operator $B$ on $\ell^{2}(V X)$ is called positivity preserving if $B_{x, y}:=$ $\left\langle\delta_{x}, B \delta_{y}\right\rangle \geq 0$ for any $x, y \in V X$. A sequence $\{v(x) \mid x \in V X\}$ is called a Perron-Frobenius weight for $B$ if it has positive entries and

$$
\sum_{y \in V X} B_{x, y} v(y)=\|B\| v(x)
$$


for any $x \in V X$.

In [8], F. Fidaleo considered BEC on graphs and showed the following two results.

Proposition 4.6. 8, Proposition 4.1.] Let $A_{X}$ be the adjacency operator of $X$ on $\ell^{2}(V X)$ and $H$ be the Hamiltonian defined by $H=\left\|A_{X}\right\| \mathbb{1}-A_{X}$. Let $\mathfrak{h}$ be a subspace of $\ell^{2}(V X)$ satisfying the following three conditions: For each $\beta>0$,

1. $e^{i t H} \mathfrak{h}=\mathfrak{h}, t \in \mathbb{R}$

2. For each entire function $f, f(H) \mathfrak{h} \subset \mathcal{D}\left(\left(e^{\beta H}-1\right)^{-1 / 2}\right)$;

3. $\sum_{x \in V X}|(f(H) u)(x)| v(x)<\infty$, and $\langle f(H) u, v\rangle=\overline{f(0)}\langle u, v\rangle$, where $v$ is a Perron-Frobenius weight for $A_{X}$.

Then for $D \geq 0$, the two-point function

$$
\varphi_{D}\left(a^{*}\left(f_{1}\right) a\left(f_{2}\right)\right)=\left\langle\left(e^{\beta H}-\mathbb{1}\right)^{-1} f_{2}, f_{1}\right\rangle_{\ell^{2}}+D\left\langle f_{2}, v\right\rangle\left\langle v, f_{1}\right\rangle
$$

satisfies the KMS condition at inverse temperature $\beta>0$ on the Weyl CCR algebra $\mathcal{W}(\mathfrak{h}, \sigma)$ with respect to the dynamics generated by the Bogoliubov transformations

$$
f \in \mathfrak{h} \mapsto e^{i t H} f, \quad t \in \mathbb{R} .
$$

By the above proposition and [14, Proposition 1.1.], we are said to be BEC occur if the case of $D>0$ and BEC does not occur if the case of $D=0$.

Theorem 4.7. 8, Theorem 4.5.] Suppose that $A_{X}$ is transient. Let $\mathfrak{h}_{1}$ be the subspace of $\ell^{2}(V X)$ defined by

$$
\mathfrak{h}_{1}=\left\{e^{i t H} \delta_{x} \mid t \in \mathbb{R}, x \in V X\right\} .
$$

Then $\mathfrak{h}_{1}$ satisfies the conditions 1, 2, and 3 in Proposition 4.6. Thus, for $\mathfrak{h}_{1}$ and any $D \geq 0$, the two-point function given in (4.26) defines KMS state on the Weyl CCR algebra $\mathcal{W}\left(\mathfrak{h}_{1}, \sigma\right)$.

We give another example of $\mathfrak{h}$. Let $\mathcal{P}(\mathbb{C})$ be the set of all polynomial functions on $\mathbb{C}$. Let $\mathfrak{h}_{2}$ be the subspace defined by

$$
\mathfrak{h}_{2}=\left\{\int_{\mathbb{R}} p(t) e^{-(t-a)^{2} / b} e^{i t H} \delta_{x} d t \mid p \in \mathcal{P}(\mathbb{C}), a \in \mathbb{R}, b>0, x \in V X\right\} .
$$

Lemma 4.8. The space $\mathfrak{h}_{2}$ satisfies the following conditions;

$$
\begin{aligned}
& 1^{\prime} . e^{i t H} \mathfrak{h}_{2}=\mathfrak{h}_{2}, t \in \mathbb{R} ; \\
& 2^{\prime} . e^{\beta H} \mathfrak{h}_{2} \subset \mathcal{D}\left(\left(e^{\beta H}-1\right)^{-1 / 2}\right) ; \\
& 3^{\prime} . \sum_{x \in V G}\left|\left(e^{\beta H} u\right)(x)\right|<\infty, \text { and }\left\langle e^{\beta H} u, v\right\rangle=\langle u, v\rangle, u \in \mathfrak{h}_{2} .
\end{aligned}
$$

Proof. The condition $1^{\prime}, e^{i t H} \mathfrak{h}_{2} \subset \mathfrak{h}_{2}$ is clear. Now we prove the condition $2^{\prime}$, $e^{\beta H} \mathfrak{h}_{2} \subset \mathcal{D}\left(\left(e^{\beta H}-\mathbb{1}\right)^{-1 / 2}\right)$. Note that $\left(e^{\beta x}-1\right)^{-1}-(\beta x)^{-1}$ is continuous on $[0, \infty)$. Thus, it enough to show that $e^{\beta H} \mathfrak{h}_{2} \subset \mathcal{D}\left(H^{-1 / 2}\right)$. Since $A_{X}$ is transient 
and $p(t) e^{-(t-a)^{2} / b}$ is a rapidly decreasing function on $\mathbb{R}$, for a generator of $\mathfrak{h}_{2}$, $\int_{\mathbb{R}} p(t) e^{-\frac{(t-a)^{2}}{b}} e^{i t H} \delta_{x} d t$, we have

$$
\begin{aligned}
& \left\langle\left(\lambda \mathbb{1}-A_{X}\right)^{-1} e^{\beta H} \int_{\mathbb{R}} p(t) e^{-\frac{(t-a)^{2}}{b}} e^{i t H} \delta_{x} d t, e^{\beta H} \int_{\mathbb{R}} p(t) e^{-\frac{(t-a)^{2}}{b}} e^{i t H} \delta_{x} d t\right\rangle \\
= & \left|\int_{\mathbb{R}} \int_{\mathbb{R}} \overline{p(t)} p(s) e^{-\frac{(t-a)^{2}}{b}} e^{-\frac{(s-a)^{2}}{b}}\left\langle\left(\lambda \mathbb{1}-A_{X}\right)^{-1} e^{\beta H} e^{i t H} \delta_{x}, e^{\beta H} e^{i s H} \delta_{x}\right\rangle d t d s\right| \\
= & \left|\int_{\mathbb{R}} \int_{\mathbb{R}} \overline{p(t)} p(s) e^{-\frac{(t-a)^{2}}{b}} e^{-\frac{(s-a)^{2}}{b}} \int_{\sigma\left(A_{X}\right)} \frac{e^{i(s-t) a} e^{2 \beta\left(\left\|A_{X}\right\| \mathbb{1}-a\right)}}{\lambda-a} d\left\langle\delta_{x}, E(a) \delta_{x}\right\rangle d t d s\right| \\
\leq & C_{1} e^{4 \beta\left\|A_{X}\right\|}\left\langle\left(\lambda \mathbb{1}-A_{X}\right)^{-1} \delta_{x}, \delta_{x}\right\rangle \nearrow C_{1} e^{2 \beta\left\|A_{X}\right\|}\left\langle\left(\left\|A_{X}\right\| \mathbb{1}-A_{X}\right)^{-1} \delta_{x}, \delta_{x}\right\rangle<\infty,
\end{aligned}
$$

where $C_{1}$ is a positive constant satisfying

$$
\int_{\mathbb{R}} \int_{\mathbb{R}}\left|\overline{p(t)} p(s) e^{-\frac{(t-a)^{2}}{b}} e^{-\frac{(s-a)^{2}}{b}}\right| d t d s<C_{1} .
$$

Next, we show that $\sup _{n \in \mathbb{N}} \sum_{x \in V \Lambda}\left|\left(e^{\beta H} u\right)(x)\right| v(x)<\infty, u \in \mathfrak{h}_{2}$, where $\Lambda_{n}$ is a finite subgraph of $X$ such that $\Lambda_{n} \nearrow X$. Let $C_{R}$ be a circle centered at the origin with radius $R>\left\|A_{X}\right\|$. We have

$$
\begin{aligned}
& \left|\left\langle e^{\beta H} \int_{\mathbb{R}} p(t) e^{-\frac{(t-a)^{2}}{b}} e^{i t H} \delta_{x} d t, \delta_{y}\right\rangle\right| \leq \int_{\mathbb{R}}|p(t)| e^{-\frac{(t-a)^{2}}{b}}\left|\left\langle e^{\beta H} e^{i t H} \delta_{x}, \delta_{y}\right\rangle\right| d t \\
= & \int_{\mathbb{R}}|p(t)| e^{-\frac{(t-a)^{2}}{b}}\left|\frac{1}{2 \pi i} \oint_{C_{R}} e^{\beta z} e^{i t z}\left\langle\left(z \mathbb{1}-A_{X}\right)^{-1} \delta_{x}, \delta_{y}\right\rangle d z\right| d t \\
\leq & R e^{\beta R} \int_{\mathbb{R}}|p(t)| e^{-\frac{(t-a)^{2}}{b}} e^{t R} d t\left\langle\left(R \mathbb{1}-A_{X}\right)^{-1} \delta_{x}, \delta_{y}\right\rangle \leq C_{2}\left\langle\left(R \mathbb{1}-A_{X}\right)^{-1} \delta_{x}, \delta_{y}\right\rangle,
\end{aligned}
$$

for any $x, y \in V X$, where $C_{2}$ is a positive constant satisfying

$$
R e^{\beta R} \int_{\mathbb{R}}|p(t)| e^{-\frac{(t-a)^{2}}{b}} e^{t R} d t<C_{2} .
$$

By the above inequality 4.32 , we get

$$
\begin{aligned}
& \sum_{y \in V \Lambda_{n}}\left|\left\langle e^{\beta H} \int_{\mathbb{R}} p(t) e^{-\frac{(t-a)^{2}}{b}} e^{i t H} \delta_{x} d t, \delta_{y}\right\rangle\right| v(y) \leq C_{2} \sum_{y \in V \Lambda_{n}}\left\langle\left(R \mathbb{1}-A_{X}\right)^{-1} \delta_{x}, \delta_{y}\right\rangle v(y) \\
= & C_{2}\left\langle\left(R \mathbb{1}-A_{X}\right)^{-1} \delta_{x}, v\left\lceil_{V \Lambda_{n}}\right\rangle=C_{2} \sum_{k=0}^{\infty} \frac{\left\langle A_{X}^{k} \delta_{x}, v\left\lceil_{V \Lambda_{n}}\right\rangle\right.}{R^{k+1}}\right. \\
\leq & C_{2}\left(R-\left\|A_{X}\right\|\right)^{-1} v(x) .
\end{aligned}
$$

Finally, we show the latter part of the condition $3^{\prime}$. For any $f \in \mathfrak{h}_{2}$, by definition of $v$,

$$
\left\langle e^{\beta H} f, v\right\rangle=\langle f, v\rangle .
$$

Thus, we are done. 
Theorem 4.9. Suppose that the adjacency operator $A_{X}$ of a graph $X$ is transient. For $D>0$, the two-point function $\varphi_{D}$ defined in 4.26 is a non-factor $K M S$ state on $\mathcal{W}\left(\mathfrak{h}_{1}, \sigma\right)$ or $\mathcal{W}\left(\mathfrak{h}_{2}, \sigma\right)$. Moreover, we have factor decomposition of $\varphi_{D}$ into extremal KMS states

$$
\varphi_{D}=\frac{1}{2 \pi} \int_{\mathbb{R}^{2}} \varphi_{s_{1}, s_{2}} e^{-\frac{s_{1}^{2}+s_{2}^{2}}{2}} d s_{1} d s_{2} .
$$

Proof. Since $\left\langle\cdot,\left(e^{\beta H}+\mathbb{1}\right)\left(e^{\beta H}-\mathbb{1}\right)^{-1} \cdot\right\rangle$ is positive definite inner product on $\mathfrak{h}_{1}$ and $\mathfrak{h}_{2}$, it suffice to show that $\langle v, f\rangle, f \in \mathfrak{h}_{1}$ or $f \in \mathfrak{h}_{2}$ is not continuous with respect to the norm $\left\langle\cdot,\left(e^{\beta H}+\mathbb{1}\right)\left(e^{\beta H}-\mathbb{1}\right)^{-1} \cdot\right\rangle$ by Theorem 4.4 and 4.5 . Let $p_{n}$ be the polynomial defined by

$$
p_{n}(x)=\sum_{k=0}^{n} \frac{(-n x)^{k}}{k !} .
$$

For any $f \in \mathfrak{h}_{1},\left(p_{n}(H)-\mathbb{1}\right) f \in \mathfrak{h}_{1}$. Put $f_{n}=\left(p_{n}(H)-\mathbb{1}\right) f$. Then

$$
\left\langle f_{n}-f,\left(e^{\beta H}+\mathbb{1}\right)\left(e^{\beta H}-\mathbb{1}\right)^{-1}\left(f_{n}-f\right)\right\rangle \rightarrow 0, \quad(n \rightarrow \infty)
$$

and

$$
\left\langle v, f_{n}\right\rangle=0
$$

for any $n \in \mathbb{N}$. Thus, we have that $\langle v, \cdot\rangle$ is not continuous.

For any $f \in \mathfrak{h}_{2}$, we put $f_{n}=p_{n}(H) f$. We can show $f_{n} \in \mathfrak{h}_{2}$. Similarly the case of $\mathfrak{h}_{1}$, we can prove the statement.

\section{Acknowledgments}

The author would like to thank Professor Taku Matsui for discussions and comments for this work.

\section{References}

[1] H. Araki, M. Shiraishi, On quasifree states of the canonical commutation relations. I, Publ. Res. Inst. Math. Sci. 7 (1971/72), 105-120.

[2] H. Araki, On quasifree states of the canonical commutation relations. II, Publ. Res. Inst. Math. Sci. 7 (1971/72), 121-152.

[3] H. Araki, S. Yamagami, On quasi-equivalence of quasifree states of the canonical commutation relations. Publ. Res. Inst. Math. Sci. 18 (1982), no. $2,703-758$

[4] O. Bratteli, D. Robinson, Operator algebras and quantum statistical mechanics I, 2nd edition (Springer, 1986.)

[5] O. Bratteli, D. Robinson, Operator algebras and quantum statistical mechanics II, 2nd edition (Springer, 1997.)

[6] F. Fidaleo, D. Guido, T. Isola, Bose-Einstein condensation on inhomogeneous amenable graphs. Infin. Dimens. Anal. Quantum Probab. Relat. Top. 14 (2011), no. 2, 149-197. 
[7] F. Fidaleo, Harmonic analysis on Cayley trees II: The Bose-Einstein condensation. Infin. Dimens. Anal. Quantum Probab. Relat. Top. 15 (2012), no. 4, 1250024, 32 pp.

[8] F. Fidaleo, Harmonic analysis on inhomogeneous amenable networks and the Bose-Einstein condensation. J. Stat. Phys. 160 (2015), no. 3, 715-759.

[9] R. Honegger, Decomposition of positive sesquilinear forms and the central decomposition of gauge-invariant quasi-free states on the Weyl-algebra. $Z$. Naturforsch. A 45 (1990), no. 1, 17-28.

[10] R. Honegger, A. Rapp, General Glauber coherent states on the Weyl algebra and their phase integrals. Phys. A 167 (1990), no. 3, 945-961.

[11] J. T. Lewis, J. V. Pulè, The equilibrium states of the free Boson gas. Comm. Math. Phys. 36 (1974), 1-18.

[12] J. Manuceau, A. Verbeure, Quasi-free states of the C.C.R.-algebra and Bogoliubov transformations. Comm. Math. Phys. 9 (1968), 293-302.

[13] J. Manuceau, F. Rocca, D. Testard, On the product form of quasi-free states. Comm. Math. Phys. 12 (1969), 43-57.

[14] T. Matsui, BEC of free bosons on networks. Infin. Dimens. Anal. Quantum Probab. Relat. Top. 9 (2006), no. 1, 1-26.

[15] J. V. Pulé, A. Verbeure, V. A. Zagrebnov, On nonhomogeneous Bose condensation. J. Math. Phys. 46 (2005), no. 8, 083301, 8 pp.

[16] F. Rocca, M. Sirugue, D. Testard, On a class of equilibrium states under the Kubo-Martin-Schwinger condition. II. Bosons. Comm. Math. Phys. 19 (1970), 119-141.

[17] B. Simon, Functional integration and quantum physics. Second edition. AMS Chelsea Publishing, Providence, RI, 2005. xiv+306 pp. ISBN: 0-8218$3582-3$

[18] A. van Daele, Quasi-equivalence of quasi-free states on the Weyl algebra. Comm. Math. Phys. 21, (1971), 171-191.

[19] S. Yamagami, Geometry of quasi-free states of CCR algebras. Internat. J. Math. 21 (2010), no. 7, 875-913.

[20] S. Yamagami, Geometry of coherent states of CCR algebras. Infin. Dimens. Anal. Quantum Probab. Relat. Top. 15 (2012), no. 2, 1250009, 9 pp. 